\title{
Classification and Diagnostic Criteria for IgG4-Related Sclerosing Cholangitis
}

\author{
Itaru Naitoh and Takahiro Nakazawa \\ Department of Gastroenterology, Nagoya City University Graduate School of Medical Sciences, Nagoya, Japan
}

\section{Article Info}

Received March 12, 2021

Revised May 6, 2021

Accepted June 14, 2021

Published online August 13, 2021

\section{Corresponding Author}

Itaru Naitoh

ORCID https://orcid.org/0000-0001-8342-886X

E-mail inaito@med.nagoya-cu.ac.jp
IgG4-related sclerosing cholangitis (IgG4-SC) can be classified into four types based on cholangiographic findings and regions of biliary stricture. This cholangiographic classification is useful to differentiate IgG4-SC from mimickers including cholangiocarcinoma, primary sclerosing cholangitis, and pancreatic cancer. Autoimmune pancreatitis (AIP) is a valuable clue for the diagnosis of IgG4-SC because the two are frequently found in association with each other. Two sets of diagnostic criteria for IgG4-SC have been proposed. In Japan, the clinical diagnostic criteria 2020 were recently developed. These clinical diagnostic criteria include narrowing of the intrahepatic and/or extrahepatic bile duct, thickening of the bile duct wall, serological findings, pathological findings, other organ involvement, and effectiveness of steroid therapy. When these criteria are applied, IgG4-SC is initially classified as associated or not associated with AIP, and cholangiographic classification is used for differential diagnosis. In most instances, IgG4-SC can be diagnosed on the basis of clinical diagnostic criteria. However, it is challenging to diagnose isolated IgG4-SC or IgG4-SC not associated with AIP. Here, we review the classification and diagnostic criteria for lgG4-SC, specifically focusing on the clinical diagnostic criteria 2020 and a large lgG4SC case series from a nationwide survey in Japan. (Gut Liver 2022;16:28-36)

Key Words: Autoimmune pancreatitis; Cholangiographic classification; Diagnostic criteria; IgG4related sclerosing cholangitis

\section{INTRODUCTION}

IgG4-related sclerosing cholangitis (IgG4-SC) is a distinct type of cholangitis of unknown pathogenesis. It is frequently associated with autoimmune pancreatitis (AIP), and is considered as a biliary involvement of IgG4-related disease (IgG4-RD) ${ }^{1-3}$ Patients with IgG4-SC present with bile duct stricture and the bile duct wall thickening. ${ }^{4-6}$ Cholangiograms of patients with IgG4-SC are similar to those of patients with cholangiocarcinoma and primary sclerosing cholangitis (PSC), and pancreatic cancer. These diseases are notable mimickers of IgG4-SC. ${ }^{4-6}$ Patients with IgG4-SC respond well to steroid therapy and positive outcomes after steroid administration. ${ }^{7,8}$ PSC, in contrast, is a progressive disease that results in liver cirrhosis. Liver transplantation is the only treatment for patients with advanced PSC. ${ }^{8-11}$ Treatment of cholangiocarcinoma and pancreatic cancer requires surgical treatment or chemo- therapy. IgG4-SC and its mimickers have different treatments and clinical outcomes. Differentiating the diseases is important for the selection of appropriate therapy. However, diagnosing IgG4-SC can be difficult. This is especially true when IgG4-SC is not associated with AIP (IgG4-SC without AIP), as well as when IgG4-SC is associated with AIP (IgG4-SC with AIP) that occurs without the typical features of AIP. ${ }^{12-14}$ The classification and diagnostic criteria for IgG4-SC are useful for differentiating it from other mimickers. ${ }^{4,6,15-18}$ Here, we review the classification and diagnostic criteria for IgG4-SC. We focus on the clinical diagnostic criteria 2020, which were developed recently in Japan, ${ }^{19}$ and a large case series of patients with IgG4-SC based on a nationwide survey conducted in Japan. ${ }^{20}$ 


\section{CLASSIFICATION OF IgG4-SC}

\section{Cholangiographic classification}

IgG4-SC can be classified into four types based on the regions of biliary stricture shown in cholangiograms and the differential diagnoses that must be considered (Fig. 1). ${ }^{15}$ Type 1 IgG4-SC shows biliary stricture only in the intrapancreatic bile duct, and should be differentiated from pancreatic cancer, cholangiocarcinoma, and chronic pancreatitis. Type 2 IgG4-SC, in which biliary stricture is diffusely distributed throughout the intra- and extrahepatic bile ducts, should be differentiated from PSC. Type 2 IgG4-SC is divided into two subtypes: 2 a, characterized by biliary stricture of the intrahepatic bile ducts with prestenotic dilation and $2 \mathrm{~b}$, characterized by reduced bile duct branches and biliary stricture of the intrahepatic bile ducts without prestenotic dilation. Type 3 IgG4-SC is characterized by biliary stricture in the hilar hepatic and intrapancreatic bile ducts. Type 4 IgG4-SC involves biliary stenoses of the bile duct only in hilar hepatic lesions. Type 3 and type 4 IgG4-SC must be discriminated from cholangiocarcinoma, particularly cholangiocarcinoma with hilar lesions. This cholangiographic classification is useful for identifying helpful diagnostic modalities and differentiating IgG4-SC from other mimickers. The classification is incorporated into the clinical diagnostic criteria $2020 .{ }^{19}$ In a recent large case series in Japan, ${ }^{20}$ type 1 was the most common cholangiogram of IgG4-SC (62.9\%) and IgG4-SC with AIP (69.9\%). However, type 1 was observed in only $23.8 \%$ of patients who had IgG4-SC without AIP, and type 4 was the most common cholangiogram in patients who had IgG4-SC without AIP (30.9\%).

\section{Association with AIP}

IgG4-SC can be classified based on the presence or absence of its association with AIP. IgG4-SC is commonly associated with AIP, such that AIP is recognized in $83 \%$ to $92 \%$ of patients with IgG4-SC., ${ }^{7,16,20}$ The association with AIP is useful in more precise diagnosis of IgG4-SC. Therefore, the clinical diagnostic criteria 2020 first classify IgG4SC based on its association with AIP. ${ }^{19}$ Instances of IgG4SC without IgG4-RD are referred to as "isolated IgG4SC."12,14,21 Diagnosing isolated IgG4-SC can be difficult. Cholangiographic findings in patients with isolated IgG4-

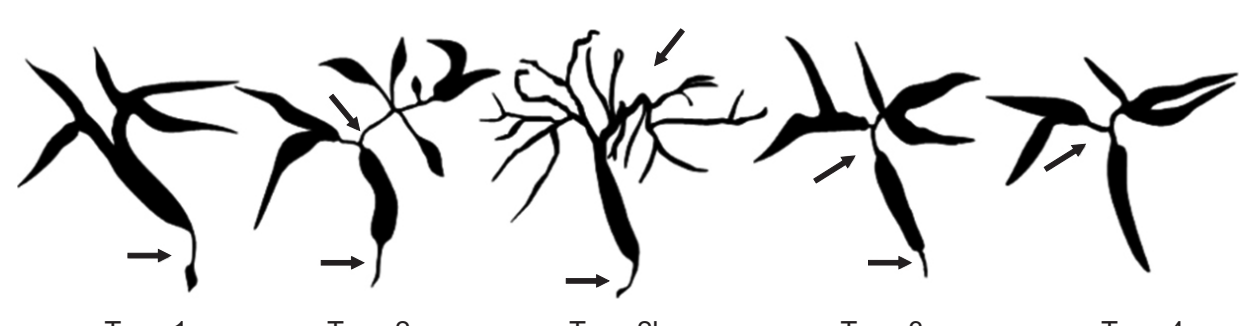

Type 1

Type 2a

Type $2 b$

Type 3

Type 4

Fig. 1. Cholangiographic classification of IgG4-related sclerosing cholangitis. Type 1: stricture is located in only the intrapancreatic bile duct. Type 2: stricture is diffusely distributed throughout the intrahepatic and extrahepatic bile ducts. Type 2a: stenosis of the intrahepatic bile ducts with prestenotic dilatation. Type $2 \mathrm{~b}$ : stenosis of the intrahepatic bile ducts without prestenotic dilatation and reduced bile duct branches. Type 3: stricture is located in both the hilar hepatic and intrapancreatic bile ducts. Type 4: stricture is located in only the hilar hepatic bile ducts. Arrows: stenosis of the bile ducts. Adapted from Nakazawa T, et al. Pancreas 2006;32:229, with permission from Wolters Kluwer Health, Inc. ${ }^{15}$

Table 1. HISORt Criteria for IgG4-Related Sclerosing Cholangitis

\begin{tabular}{|c|c|}
\hline Feature & Characteristics \\
\hline Histology of bile duct & $\begin{array}{l}\text { Lymphoplasmacytic sclerosing cholangitis on resection specimens (lymphoplasmacytic infiltrate with >10 IgG4- } \\
\text { positive cells/HPF within and around bile ducts with associated obliterative phlebitis and storiform fibrosis) }\end{array}$ \\
\hline Imaging of bile duct & $\begin{array}{l}\text { One or more strictures involving intrahepatic, proximal extrahepatic, or intrapancreatic bile ducts } \\
\text { Fleeting/migrating biliary strictures }\end{array}$ \\
\hline Serology & Increased levels of serum IgG4 \\
\hline Other organ involvement & $\begin{array}{l}\text { Pancreas: classic features of autoimmune pancreatitis on imaging or histology; suggestive pancreatic imaging find- } \\
\text { ings: focal pancreatic mass/enlargement without pancreatic duct dilatation, multiple pancreatic masses, focal } \\
\text { pancreatic duct stricture without upstream dilatation, pancreatic atrophy } \\
\text { Retroperitoneal fibrosis } \\
\text { Renal lesions: single or multiple parenchymal low-attenuation lesions (round, wedge-shaped, or diffuse patchy) } \\
\text { Salivary/lacrimal gland enlargement }\end{array}$ \\
\hline Response to steroid therapy & Normalization of liver enzyme levels or resolution of stricture \\
\hline
\end{tabular}

HPF, high-power field.

Adapted from Ghazale A, et al. Gastroenterology 2008;134:706-715, with permission from Elsevier. ${ }^{16}$ 
SC most frequently involve type 4 IgG4-SC. ${ }^{7,20,22}$ Discriminating isolated type $4 \mathrm{IgG} 4-\mathrm{SC}$ from cholangiocarcinoma with hilar lesion is challenging.

\section{DIAGNOSTIC CRITERIA FOR IgG4-SC}

Two sets of diagnostic criteria for IgG4-SC have been proposed to date. ${ }^{4,16}$ The HISORt criteria were proposed by a US group (Table 1$)^{16}$ and are based on histology, imaging, serology, other organ involvement (OOI), and response to steroid therapy. The HISORt criteria were originally developed for diagnosis of AIP and later adapted for diagnosis of IgG4-SC. In 2012, a Japanese group proposed the clinical diagnostic criteria 2012 (Table 2). ${ }^{4}$ The clinical diagnostic criteria 2012 include four criteria: characteristic biliary imaging findings, elevation of serum IgG4 concentrations, coexistence with other IgG4-RDs, and characteristic histopathological features. The effectiveness of steroid therapy is an optional additional diagnostic criterion for confirming IgG4-SC. In 2020, a Japanese group proposed new clinical diagnostic criteria based on a revision of the clinical diagnostic criteria 2012 (Table 3). ${ }^{19}$ These clinical diagnostic criteria 2020 include six criteria: narrowing of the intrahepatic and/or extrahepatic bile duct, thickening of the bile duct wall, serological findings, pathological findings, OOI, and effectiveness of steroid therapy. In the clinical diagnostic criteria 2020, the patient is initially classified as "with AIP" or "without AIP." Next, cholangiographic classification is used to make a differential diagnosis. In this section, we review each criterion in the clinical diagnostic criteria 2020.

\section{Narrowing of the bile duct}

Endoscopic retrograde cholangiopancreatography (ERCP) and magnetic resonance cholangiopancreatography (MRCP) are used to evaluate narrowing of the bile duct. Diffuse and segmental strictures of the bile ducts are revealed by these modalities. Relatively long strictures are characteristic features of IgG4-SC. Intrapancreatic biliary strictures are also characteristic, and are the most common feature of cholangiograms in IgG4-SC. In contrast, band-like strictures (1 to $2 \mathrm{~mm}$ ), beaded or prunedtree appearance, and diverticulum-like outpouching are characteristic features of PSC. ERCP is a useful procedure for distinguishing type 2 IgG4-SC and PSC because these characteristics can be visualized (Fig. 2). ${ }^{23,24}$ Percutaneous transhepatic cholangiography is also useful for evaluating biliary stenoses in patients with IgG4-SC. ${ }^{6,19}$ MRCP is not superior to ERCP for evaluating biliary strictures, although additional information about the pancreatic duct can be obtained without the potential for ERCP-related pancreatitis. A recent large case series in Japan found that ERCP and MRCP had similar visualization rates for bandlike strictures, segmental strictures, long strictures, beaded appearance, and pruned-tree appearance in patients with IgG4-SC. ${ }^{20}$ Therefore, MRCP is an option for evaluating biliary strictures when images good enough for detailed evaluation of the biliary tract can be obtained. MRCP is included in the clinical diagnostic criteria 2020 as a method for evaluating the stricture of the bile duct because of a recent improvement in image quality and reduction in invasiveness.

Table 2. Clinical Diagnostic Criteria of IgG4-Related Sclerosing Cholangitis 2012

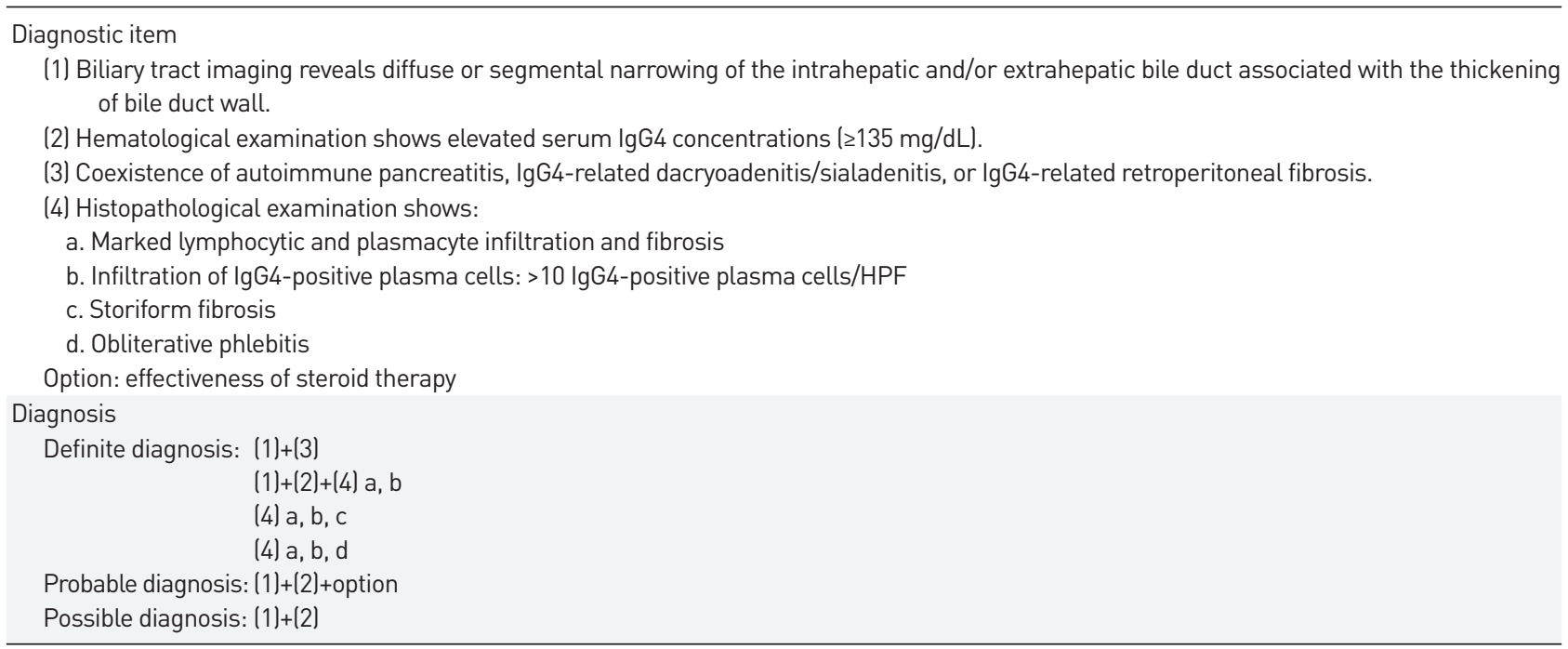

HPF, high-power field.

Adapted from Ohara H, et al. J Hepatobiliary Pancreat Sci 2012;19:536-542, with permission from John Wiley and Sons. ${ }^{4}$ 


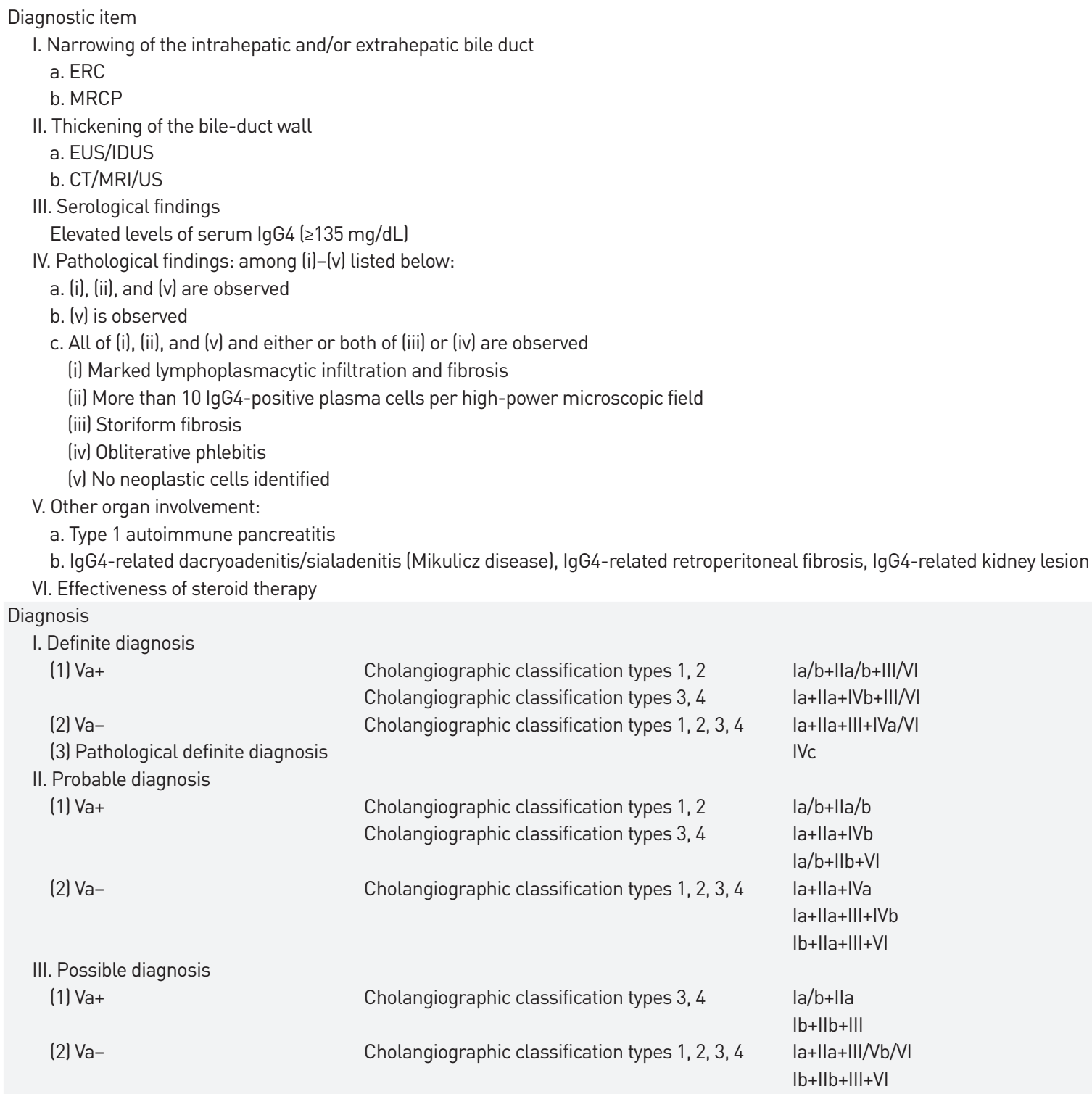

(1) Va+

(2) Va-

(3) Pathological definite diagnosis

II. Probable diagnosis

(1) Va+

(2) Va-

III. Possible diagnosis

(1) $\mathrm{Va}+$

(2) Va-

Cholangiographic classification types 1, 2

Cholangiographic classification types 3,4

Cholangiographic classification types 1, 2, 3, 4

Cholangiographic classification types 1,2

Cholangiographic classification types 3, 4

Cholangiographic classification types 1, 2, 3, 4

Cholangiographic classification types 3, 4

Cholangiographic classification types 1, 2, 3, 4

$|\mathrm{a} / \mathrm{b}+| \mathrm{la} / \mathrm{b}+\mid \mathrm{II} / \mathrm{Vl}$

$\mathrm{la}+\mathrm{Ila}+|\mathrm{Vb}+| \mathrm{II} / \mathrm{VI}$

$|\mathrm{a}+| \mathrm{Ia}+||++\mid \mathrm{Va} / \mathrm{VI}$

IVc

$|a / b+| l a / b$

$\mathrm{la}+\mathrm{Il} \mathrm{a}+\mathrm{IVb}$

$|a / b+| l b+V \mid$

$l a+I l a+I V a$

$|a+I l a+I I|+I V b$

$|b+||a+|||+V \mid$

$\mathrm{la} / \mathrm{b}+1 \mathrm{la}$

$|b+| l b+|l|$

$|\mathrm{a}+| \mathrm{la}+\mid \mathrm{II} / \mathrm{Vb} / \mathrm{Vl}$

$|b+||b+|||+V \mid$

ERC, endoscopic retrograde cholangiography; MRCP, magnetic resonance cholangiopancreatography; EUS, endoscopic ultrasonography; IDUS, intraductal ultrasonography; CT, computed tomography; MRI, magnetic resonance imaging; US, ultrasonography.

Adapted from Nakazawa T, et al. J Hepatobiliary Pancreat Sci 2021;28:235-242, with permission from John Wiley and Sons. ${ }^{19}$

\section{Thickening of the bile duct wall}

Ultrasonography (US), computed tomography (CT), magnetic resonance imaging (MRI), endoscopic ultrasonography (EUS), and intraductal ultrasonography (IDUS) are used to evaluate thickening of the bile duct wall. Circular and symmetrical thickening of the bile duct wall with a smooth inner margin is a characteristic feature of IgG4SC. In cholangiograms, the thickening of the bile duct wall is observed at the stricture and at non-stricture lesions. US, $\mathrm{CT}$, and MRI can detect thickening of the bile duct wall, which is a clue in the diagnosis of IgG4-SC. Thickening of the bile duct wall was detected on US (81.2\%), CT (71.4\%), and MRI (65.9\%) in the recent large case series in Japan. ${ }^{20}$ However, it is challenging to detect differences in the details of bile duct wall thickening in IgG4-SC, compared with other diseases, when using these modalities. EUS and IDUS are useful for differentiating IgG4-SC from other mimickers because they provide higher resolution images of the bile duct wall, compared with other modalities. Furthermore, slight changes of bile duct wall thickening can be observed clearly when using IDUS. IDUS findings in patients with IgG4-SC include circular and symmetrical wall thickening, smooth inner and outer margin, and homogeneous internal echo in the stenotic area. ${ }^{25-29}$ In contrast, 


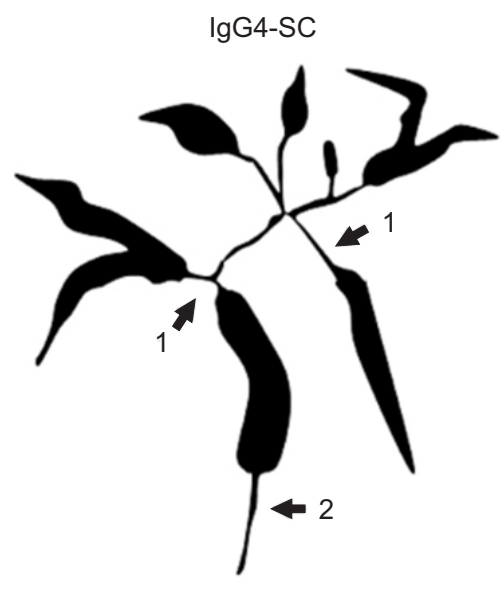

1. Dilation after confluent stricture

2. Stricture of lower common bile duct

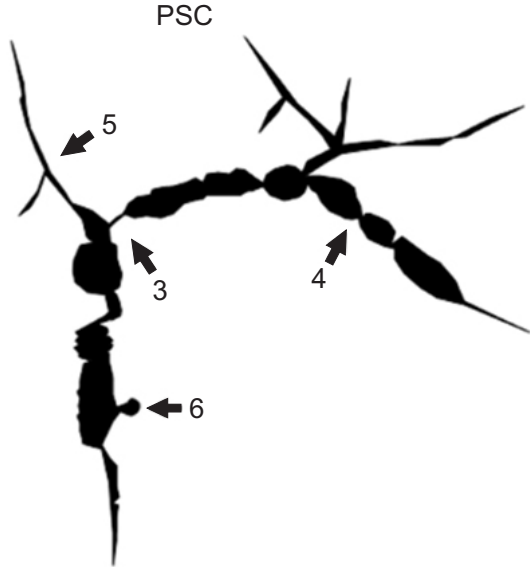

3. Band-like stricture

4. Beaded appearance

5. Pruned-tree appearance

6 . Diverticulum-like outpouching
Fig. 2. Characteristic features of cholangiogram between IgG4-related sclerosing cholangitis (lgG4-SC) and primary sclerosing cholangitis (PSC) on endoscopic retrograde cholangiography.
IgG4-SC

Symmetric

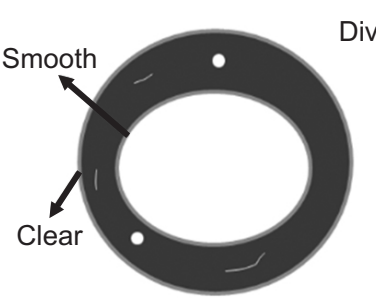

Homogeneous

Preservation of three layers
PSC

Asymmetric

Diverticulum-like outpouching

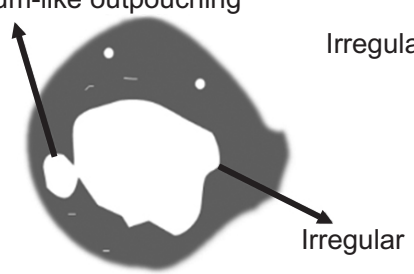

Heterogeneous

Disappearance of three layers
Cholangiocarcinoma

Asymmetric

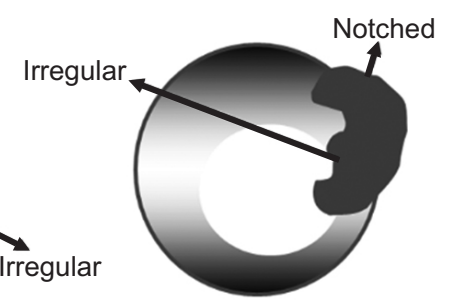

Heterogeneous
Disappearance of three layers
Fig. 3. Intraductal ultrasonographic findings in the biliary stricture among IgG4-SC, PSC, and cholangiocarcinoma.

IgG4-SC, IgG4-related sclerosing cholangitis; PSC, primary sclerosing cholangitis. Naitoh I, et al. J Gastroenterol Hepatol 2015;30:1104-1109, with permission from John Wiley and Sons. ${ }^{30}$ typical IDUS findings in patients with PSC are circular and asymmetrical wall thickening, irregular inner margin and unclear outer margin, diverticulum-like outpouching, heterogeneous internal echo, and the disappearance of three layers. ${ }^{30}$ IDUS findings at the stricture site are distinct and are useful for differentiating IgG4-SC from PSC and cholangiocarcinoma (Fig. 3). Furthermore, the most characteristic IDUS finding in diagnoses of IgG4-SC is the wall thickening of the bile duct which appears normal on a cholangiogram. The wall thickening of bile duct spreads continuously from the distal common bile duct to the hilar lesion in most patients with IgG4-SC. ${ }^{25,27,30}$ A recent large case series in Japan revealed that bile duct wall thickening at non-stricture areas was present significantly more frequently in IDUS than in EUS ( $80.9 \%$ vs $73.8 \%, \mathrm{p}=0.045){ }^{20}$ IDUS findings at the non-stricture site are useful for differentiating IgG4-SC from cholangiocarcinoma because wall thickening of bile duct at the non-stricture site is not observed in cholangiocarcinoma.

\section{Serum IgG4 level}

Serum IgG4 levels are elevated in patients with IgG4-SC, and the serum IgG4 level is reported to be a useful marker in the differential diagnosis for IgG4-SC. Serum IgG4 levels $\geq 135 \mathrm{mg} / \mathrm{dL}$ are a serological criterion in the diagnostic criteria for IgG4-SC. ${ }^{4}$ However, elevated serum IgG4levels are also observed in other mimickers of IgG4-SC, such as cholangiocarcinoma, PSC, and pancreatic cancer. Only a few studies have reported on serum IgG4 levels in patients with IgG4-SC. ${ }^{12,22,31}$ A Japanese multicenter study found that a serum IgG4 level of $135 \mathrm{mg} / \mathrm{dL}$ was useful to differentiate IgG4-SC from PSC and pancreatic cancer. ${ }^{22}$ Study in the United States revealed that 13.5\% (17/126) of patients with cholangiocarcinoma had elevated serum IgG4 levels and 3.2\% (4/126) of those had a greater than 2 -fold increase. ${ }^{31}$ They concluded that a 2 -fold increase might not reliably distinguish IgG4-SC from cholangiocarcinoma, and determined that a cutoff of 4 -fold greater than the upper limit of normal was specific for IgG4-SC. Study of Europe revealed that elevated serum IgG4 levels were observed in $15 \%$ of 310 patients with PSC. ${ }^{32}$ They also 
proposed a new diagnostic algorithm based on IgG4/IgG1 ratio to distinguish IgG4-SC from PSC. Previous studies reported that lower serum IgG4 levels were observed in patients with isolated IgG4-SC. ${ }^{12-14,33}$ However, serum IgG4 levels did not differ between IgG4-SC associated with and without AIP in the large Japanese case series. ${ }^{20}$ In that study, the median serum IgG4 level was $682 \mathrm{mg} / \mathrm{dL}$ in patients with IgG4-SC who had type 4 strictures. This type of cholangiogram is most difficult to differentiate from cholangiocarcinoma.

\section{Other organ involvement}

AIP is most frequently associated with IgG4-SC, and is found in $83 \%$ to $92 \%$ of patients with IgG4-SC. ${ }^{7,6,20}$ This association with AIP is an important clue in the diagnosis of IgG4-SC. IgG4-RDs other than AIP are also associated with IgG4-SC. Examples of OOI include IgG4-related dacryoadenitis/sialadenitis, pulmonary lesions, retroperitoneal fibrosis, kidney lesions, vascular lesions, and lymph node lesions. Four IgG4-RDs such as type 1 AIP, IgG4-related dacryoadenitis/sialadenitis, IgG4-related retroperitoneal fibrosis, and IgG4-related kidney lesions are incorporated into OOIs in the clinical diagnostic criteria 2020. The recent large case series in Japan found that IgG4-SC with AIP and IgG4-SC without AIP involved similar rates of other types of IgG4-related OOI. The overall incidence of all types of IgG4-related OOI in the clinical diagnostic criteria 2020 was $18.5 \%$ in IgG4-SC without AIP. ${ }^{20}$ Consideration of IgG4-related OOIs is useful in diagnosing both IgG4-SC with and without AIP. IgG4-SC, unlike PSC, is rarely associated with inflammatory bowel diseases. ${ }^{7,916}$

\section{Histopathological examination}

Transmural marked lymphoplasmacytic infiltration and fibrosis in the intra- and extrahepatic large bile duct are histopathological findings of IgG4-SC. Storiform fibrosis and obliterative phlebitis are important findings for the diagnosis, as in other IgG4-RDs. Infiltration of abundant IgG4-positive cells is a characteristic histopathological finding of IgG4-SC. However, the infiltration of IgG4positive cells is not specific to IgG4-SC because it is also observed in PSC or cholangiocarcinoma. Infiltration of $>10$ IgG4-positive plasma cells/high-power field (HPF) is one of the histopathological findings of IgG4-SC. More than 50 IgG4-positive plasma cells/HPF are usually observed in resected specimens, and an IgG4/IgG-positive cell ratio $>40 \%$ is a characteristic histological finding in IgG4-SC.

Bile duct biopsy and cytology are useful for the diagnosis of biliary strictures. However, making a definitive diagnosis of IgG4-SC by means of bile duct biopsy is often difficult because the epithelium of bile duct is frequently histologically normal in patients with IgG4-SC. Further-

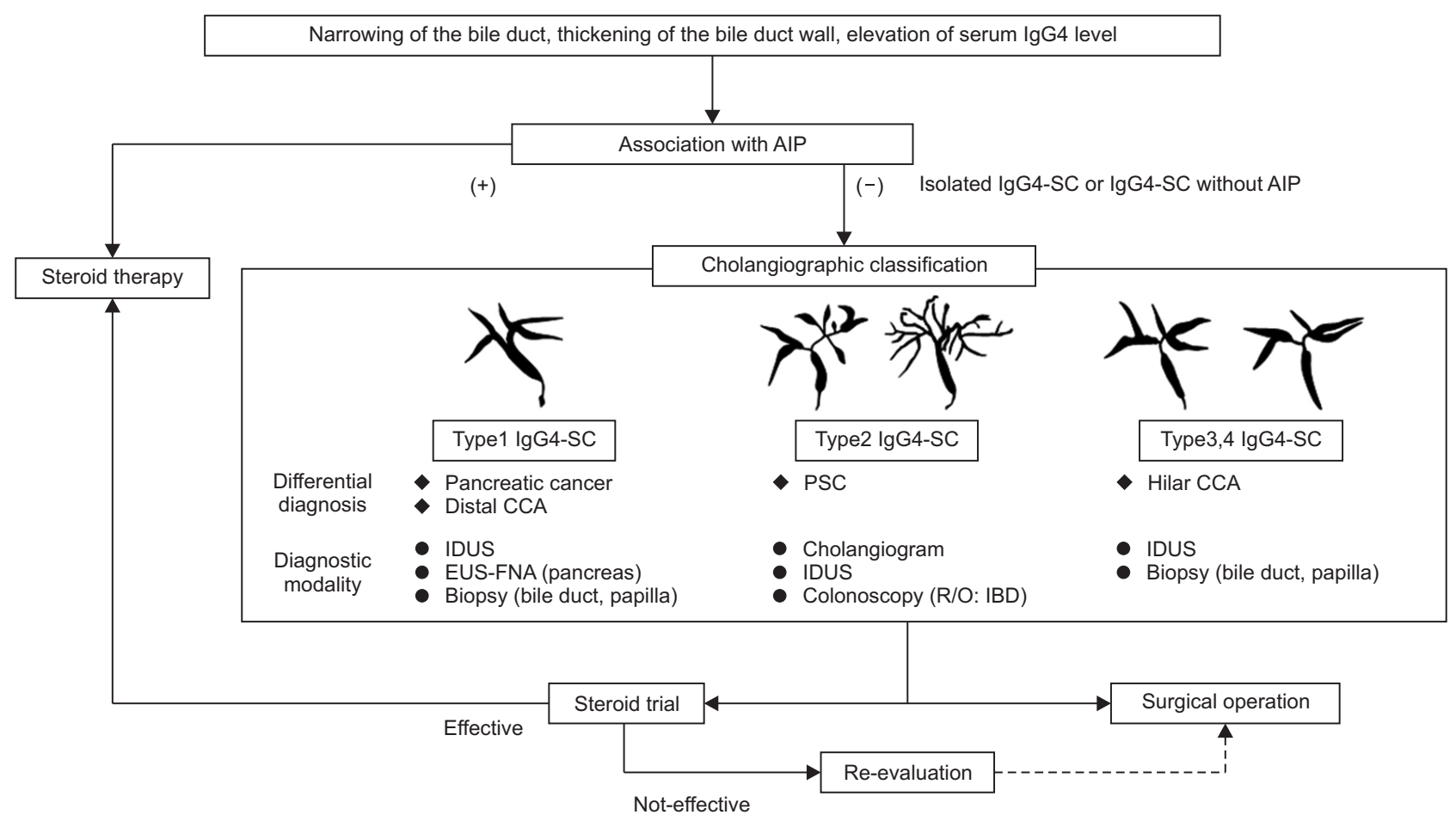

Fig. 4. Algorithm of the diagnosis and management of IgG4-SC.

IgG4-SC, IgG4-related sclerosing cholangitis; PSC, primary sclerosing cholangitis; AIP, autoimmune pancreatitis; CCA, cholangiocarcinoma; EUSFNA, endoscopic ultrasound-guided fine-needle aspiration; IBD, inflammatory bowel disease; IDUS, intraductal ultrasonography; R/O, rule out. 
more, biopsy samples containing bile duct stroma are necessary to make a diagnosis based on histopathology. The reported sensitivity of bile duct biopsy with IgG4 immunostaining ranges from $0 \%$ to $88 \%,{ }^{16,25,34,35}$ but is generally low. We consider bile duct biopsy or cytology to be important for excluding malignant biliary strictures, such as cholangiocarcinoma, although these procedures are not useful for making a definitive diagnosis of IgG4-SC.

Biopsy of duodenal papilla with IgG4-immunostaining has been a useful supplemental method for diagnosis of IgG4-SC. The reported sensitivity of duodenal papilla biopsy with IgG4 immunostaining ranges from $52 \%$ to $80 \%{ }^{27,35-39}$ In the international consensus diagnostic criteria, biopsy of the duodenal papilla is optional for the diagnosis of AIP. ${ }^{40}$ Biopsy of the duodenal papilla may be easier and more reliable, compared with bile duct biopsy, and is reportedly useful for differentiating IgG4-SC from PSC. ${ }^{37}$ Histopathological findings from biopsy of the duodenum papilla with IgG4-immunostaining are useful as a supplemental method for diagnosis of IgG4-SC.

\section{Effectiveness of steroid therapy}

Steroid therapy is the standard treatment for IgG4-SC. We usually conduct biliary drainage to prevent cholangitis after we perform ERCP-related procedures such as biliary biopsy/cytology and IDUS to differentiate IgG4-SC from other mimickers. However, biliary drainage before steroid therapy is not essential in definitive IgG4-SC cases which ERCP-related procedures are not necessary. According to a nationwide survey in Japan, steroid therapy is administered in $88 \%$ of patients with IgG4-SC and the remission rate after the administration of steroid is $90 \%{ }^{7}$ Histopathological evidence supporting a diagnosis of IgG4-SC should be obtained before a steroid trial. After 2 weeks of steroid administration, the effectiveness of steroid therapy should be evaluated. Imaging findings from US, CT, MRI, and MRCP/ERCP, not symptoms or blood tests, should be used to confirm resolution. The possibility of malignancy should be re-evaluated when steroid treatment is ineffective.

\section{ALGORITHM FOR DIAGNOSIS AND MANAGEMENT OF IgG4-SC}

We show the algorithm for the diagnosis and management of IgG4-SC (Fig. 4). We diagnose IgG4-SC on the basis of IgG4-SC2020. We suspect IgG4-SC when narrowing of the bile duct, thickening of the bile duct wall, elevation of serum IgG4 level are observed. Next, we evaluate the association with AIP because the association with AIP is important clue. When the association with AIP is present and the case is fulfilled in IgG4-SC2020, we administer steroid therapy. In the case not associated with AIP, we classify the cholangiogram into four types on the basis of the cholangiographic classification because the differential diagnosis is challenging. Cholangiocarcinoma and PSC, and pancreatic cancer are important mimickers which should be differentiated. The useful modalities for the differential diagnosis are ERCP, IDUS, bile duct biopsy, duodenal papilla biopsy, EUS-fine needle aspiration, and colonoscopy. The differential diagnosis and useful modality are different in each type of cholangiogram. When these modalities do not show characteristic findings or steroid trial is not effective, re-evaluation including pathological examination or surgical operation are considered. Surgical operation is the indication when malignant disease is confirmed or cannot be ruled out after careful re-evaluation.

\section{CONCLUSIONS}

Here, we have reviewed the classification and diagnostic criteria for IgG4-SC. The association with AIP is an important clue in the diagnosis of IgG4-SC. Cholangiographic classification is useful for differentiating IgG4-SC from other mimickers, and is incorporated into the clinical diagnostic criteria 2020. A precise diagnose of IgG4-SC can be made in most instances on the basis of clinical diagnostic criteria. However, diagnosis of isolated IgG4-SC or IgG4$\mathrm{SC}$ without AIP can be challenging. We expect that basic research will clarify the etiology and pathogenesis of IgG4SC, and that novel diagnostic modalities will be developed in the future.

\section{CONFLICTS OF INTEREST}

No potential conflict of interest relevant to this article was reported.

\section{ORCID}

Itaru Naitoh https://orcid.org/0000-0001-8342-886X Takahiro Nakazawa https://orcid.org/0000-0002-6321-5995

\section{REFERENCES}

1. Kamisawa T, Funata N, Hayashi Y, et al. A new clinicopathological entity of IgG4-related autoimmune disease. J Gastro- 
enterol 2003;38:982-984.

2. Umehara H, Okazaki K, Masaki Y, et al. Comprehensive diagnostic criteria for IgG4-related disease (IgG4-RD), 2011. Mod Rheumatol 2012;22:21-30.

3. Kamisawa T, Zen Y, Pillai S, Stone JH. IgG4-related disease. Lancet 2015;385:1460-1471.

4. Ohara H, Okazaki K, Tsubouchi $\mathrm{H}$, et al. Clinical diagnostic criteria of IgG4-related sclerosing cholangitis 2012. J Hepatobiliary Pancreat Sci 2012;19:536-542.

5. Nakazawa T, Shimizu S, Naitoh I. IgG4-related sclerosing cholangitis. Semin Liver Dis 2016;36:216-228.

6. Kamisawa T, Nakazawa T, Tazuma S, et al. Clinical practice guidelines for IgG4-related sclerosing cholangitis. J Hepatobiliary Pancreat Sci 2019;26:9-42.

7. Tanaka A, Tazuma S, Okazaki K, et al. Clinical features, response to treatment, and outcomes of IgG4-related sclerosing cholangitis. Clin Gastroenterol Hepatol 2017;15:920-926.

8. Tanaka A. IgG4-related sclerosing cholangitis and primary sclerosing cholangitis. Gut Liver 2019;13:300-307.

9. Tanaka A, Tazuma S, Okazaki K, Tsubouchi H, Inui K, Takikawa H. Nationwide survey for primary sclerosing cholangitis and IgG4-related sclerosing cholangitis in Japan. J Hepatobiliary Pancreat Sci 2014;21:43-50.

10. Isayama H, Tazuma S, Kokudo N, et al. Clinical guidelines for primary sclerosing cholangitis 2017. J Gastroenterol 2018;53:1006-1034

11. Chapman MH, Thorburn D, Hirschfield GM, et al. British Society of Gastroenterology and UK-PSC guidelines for the diagnosis and management of primary sclerosing cholangitis. Gut 2019;68:1356-1378.

12. Graham RP, Smyrk TC, Chari ST, Takahashi N, Zhang L. Isolated IgG4-related sclerosing cholangitis: a report of 9 cases. Hum Pathol 2014;45:1722-1729.

13. Nakazawa T, Ikeda Y, Kawaguchi Y, et al. Isolated intrapancreatic IgG4-related sclerosing cholangitis. World J Gastroenterol 2015;21:1334-1343.

14. Takagi Y, Kubota K, Takayanagi T, et al. Clinical features of isolated proximal-type immunoglobulin G4-related sclerosing cholangitis. Dig Endosc 2019;31:422-430.

15. Nakazawa T, Ohara H, Sano H, Ando T, Joh T. Schematic classification of sclerosing cholangitis with autoimmune pancreatitis by cholangiography. Pancreas 2006;32:229.

16. Ghazale A, Chari ST, Zhang L, et al. Immunoglobulin G4-associated cholangitis: clinical profile and response to therapy. Gastroenterology 2008;134:706-715.

17. Nakazawa T, Naitoh I, Hayashi K, et al. Diagnostic criteria for IgG4-related sclerosing cholangitis based on cholangiographic classification. J Gastroenterol 2012;47:79-87.

18. Nakazawa T, Naitoh I, Hayashi K, Miyabe K, Simizu S, Joh T. Diagnosis of IgG4-related sclerosing cholangitis. World J Gastroenterol 2013;19:7661-7670.
19. Nakazawa T, Kamisawa T, Okazaki K, et al. Clinical diagnostic criteria for IgG4-related sclerosing cholangitis 2020: (revision of the clinical diagnostic criteria for IgG4-related sclerosing cholangitis 2012). J Hepatobiliary Pancreat Sci 2021;28:235-242.

20. Naitoh I, Kamisawa T, Tanaka A, et al. Clinical characteristics of immunoglobulin IgG4-related sclerosing cholangitis: comparison of cases with and without autoimmune pancreatitis in a large cohort. Dig Liver Dis 202;53:1308-1314.

21. Bochatay L, Majno P, Giostra E, Frossard JL. Isolated liver hilar infiltration by IgG4 inflammation mimicking cholangiocarcinoma. Case Rep Gastroenterol 2016;10:512-517.

22. Ohara H, Nakazawa T, Kawa S, et al. Establishment of a serum IgG4 cut-off value for the differential diagnosis of IgG4related sclerosing cholangitis: a Japanese cohort. J Gastroenterol Hepatol 2013;28:1247-1251.

23. Nakazawa T, Ohara H, Sano H, et al. Cholangiography can discriminate sclerosing cholangitis with autoimmune pancreatitis from primary sclerosing cholangitis. Gastrointest Endosc 2004;60:937-944.

24. Nishino T, Oyama H, Hashimoto E, et al. Clinicopathological differentiation between sclerosing cholangitis with autoimmune pancreatitis and primary sclerosing cholangitis. J Gastroenterol 2007;42:550-559.

25. Naitoh I, Nakazawa T, Ohara H, et al. Endoscopic transpapillary intraductal ultrasonography and biopsy in the diagnosis of IgG4-related sclerosing cholangitis. J Gastroenterol 2009;44:1147-1155.

26. Kubota K, Kato S, Uchiyama T, et al. Discrimination between sclerosing cholangitis-associated autoimmune pancreatitis and primary sclerosing cholangitis, cancer using intraductal ultrasonography. Dig Endosc 2011;23:10-16.

27. Moon SH, Kim MH. The role of endoscopy in the diagnosis of autoimmune pancreatitis. Gastrointest Endosc 2012;76:645-656.

28. Kamisawa T, Ohara H, Kim MH, Kanno A, Okazaki K, Fujita N. Role of endoscopy in the diagnosis of autoimmune pancreatitis and immunoglobulin G4-related sclerosing cholangitis. Dig Endosc 2014;26:627-635.

29. Kanno A, Masamune A, Shimosegawa T. Endoscopic approaches for the diagnosis of autoimmune pancreatitis. Dig Endosc 2015;27:250-258.

30. Naitoh I, Nakazawa T, Hayashi K, et al. Comparison of intraductal ultrasonography findings between primary sclerosing cholangitis and IgG4-related sclerosing cholangitis. J Gastroenterol Hepatol 2015;30:1104-1109.

31. Oseini AM, Chaiteerakij R, Shire AM, et al. Utility of serum immunoglobulin G4 in distinguishing immunoglobulin G4associated cholangitis from cholangiocarcinoma. Hepatology 2011;54:940-948.

32. Boonstra K, Culver EL, de Buy Wenniger LM, et al. Serum 
immunoglobulin G4 and immunoglobulin G1 for distinguishing immunoglobulin G4-associated cholangitis from primary sclerosing cholangitis. Hepatology 2014;59:19541963.

33. Hamano H, Kawa S, Uehara T, et al. Immunoglobulin G4-related lymphoplasmacytic sclerosing cholangitis that mimics infiltrating hilar cholangiocarcinoma: part of a spectrum of autoimmune pancreatitis? Gastrointest Endosc 2005;62:152157.

34. Hirano K, Fukushima N, Tada M, et al. Diagnostic utility of biopsy specimens for autoimmune pancreatitis. J Gastroenterol 2009;44:765-773.

35. Kawakami H, Zen Y, Kuwatani M, et al. IgG4-related sclerosing cholangitis and autoimmune pancreatitis: histological assessment of biopsies from Vater's ampulla and the bile duct. J Gastroenterol Hepatol 2010;25:1648-1655.

36. Kamisawa T, Tu Y, Egawa N, Tsuruta K, Okamoto A. A new diagnostic endoscopic tool for autoimmune pancreatitis.
Gastrointest Endosc 2008;68:358-361.

37. Kubota K, Kato S, Akiyama T, et al. Differentiating sclerosing cholangitis caused by autoimmune pancreatitis and primary sclerosing cholangitis according to endoscopic duodenal papillary features. Gastrointest Endosc 2008;68:1204-1208.

38. Yamashita H, Naitoh I, Nakazawa T, et al. A comparison of the diagnostic efficacy in type 1 autoimmune pancreatitis based on biopsy specimens from various organs. Pancreatology 2014;14:186-192.

39. Moon SH, Kim MH, Park DH, et al. IgG4 immunostaining of duodenal papillary biopsy specimens may be useful for supporting a diagnosis of autoimmune pancreatitis. Gastrointest Endosc 2010;71:960-966.

40. Shimosegawa T, Chari ST, Frulloni L, et al. International consensus diagnostic criteria for autoimmune pancreatitis: guidelines of the International Association of Pancreatology. Pancreas 2011;40:352-358. 